\title{
Cause and Effect Relationship in Yield and Its Attributing Traits in Early Segregating Generations of Mustard Crosses under Terai Agro-Climatic Zone of West Bengal, India
}

\author{
Suvendu Kumar Roy ${ }^{1 *}$, Lakshmi Hijam ${ }^{1}$, Moumita Chakraborty ${ }^{1}$, Nagnathwar Vishal \\ Ashokappa ${ }^{1}$, Sanghamitra Rout ${ }^{1}$, Vinod Ashok Kale ${ }^{1}$, Bijaya Sur ${ }^{1}$, Bilin Maying ${ }^{1}$, \\ Aparajita Das ${ }^{1}$, Abhijit Kundu ${ }^{2}$, Rupsanatan Mandal ${ }^{3}$ and Hossain Ali Mondal ${ }^{3}$ \\ ${ }^{1}$ Department of Genetics and Plant Breeding, Faculty of Agriculture, Uttar Banga Krishi \\ Viswavidyalaya, Pundibari, Cooch Behar, West Bengal, India \\ ${ }^{2}$ All India Network Project on Jute and Allied Fibres, Directorate of Research, Uttar Banga \\ Krishi Viswavidyalaya, Pundibari, Cooch Behar, West Bengal, India \\ ${ }^{3}$ Regional Research Station (Terai Zone), Uttar Banga Krishi Viswavidyalaya, Pundibari, \\ Cooch Behar, West Bengal, India \\ *Corresponding author
}

\section{A B S T R A C T}

In $\mathrm{F}_{3}$ population except seeds per siliqua and 100 seed weight the 15 crosses differed significantly for all the other nine characters, however in $\mathrm{F}_{4}$ population the 15 crosses differed significantly for all the characters. Pusa Bahar $\times$ Rajasthan local selection -1

\section{Keywords}

Mustard, Correlation, Heritability, Path analysis, Direct effect, Indirect effect

Article Info

Accepted:

04 February 2018

Available Online:

10 March 2018 (13.62) was the highest performer in $F_{3}$ generation and in $F_{4}$ Rajasthan local selection $1 \times$ Pusa Barani (12.53) was the highest yielder. High $\mathrm{h}^{2}$ and GA were found for height upto first fruiting branch and seed yield per plant in both $F_{3}$ and $F_{4}$ and GA were found for height upto first fruiting branch and seed yield per plant in both $F_{3}$ and $F_{4}$ generations. At genotypic level seed yield per plant was positively associated with the plant height, days to physiological maturity, secondary branches per plant and 100 seed weight in $\mathrm{F}_{3}$ generation and primary branches per plant in $\mathrm{F}_{4}$ generation. At phenotypic level, seed yield per plant was positively associated with plant height, days to physiological maturity and secondary branches per plant and positive association and high direct effect on seed yield per plant was exhibited by plant height and secondary branches per plant in $F_{3}$ generation and in $F_{4}$ generation primary branches per plant was positively associated with seed yield per plant although it had negative direct effect on seed yield, due to its better performance through days to physiological maturity, secondary branches per plant and total chlorophyll content and indirect selection for seed yield improvement in $F_{4}$ generation is possible for the present set of mustard crosses.

\section{Introduction}

Rapeseed-mustard contributes $27 \%$ of the total oilseed production in India accounting for about $14 \%$ of world production and $22.5 \%$ of world area under rapeseed mustard. The genus Brassica comprises of six species ( $B$. campestris, B. oleraceae, B. juncea, B. nigra, 
B. napus and $B$. carinata). Among them the first three species are elementary and diploids with $2 n=16,18$ and 20 and the other three are tetraploids with chromosome members $2 n=34$, 36 and 38. The edible oil is obtained from $B$. napus, $B$. juncea and B. campestris. Oleiferous Brassicas cultivated in India are divided into three groups: rai (mustard), sarson (colza) and toria (rape). Information on the nature and magnitude of variability present in the existing material and association among the various morphological characters is a pre-requisite for any breeding programme to be initiated by the breeder for higher yields. However, seed yield, a complex character is usually controlled by non-additive gene actions and it is not only influenced by number of other morphological characters which are governed by a large number of genes, but also by environment to a great extent. Thereby, the heritable variation creates difficulty in a selection programme. Therefore, it is necessary to partition the overall variability into heritable and non-heritable components, which enables the breeders to adopt suitable breeding procedure for further improvement of genetic stocks. Mutual association of plant characters which is determined by correlation coefficient is useful for indirect selection. This further permits evaluation of relative influence of various components of yield. The path coefficient analysis proposed by Wright (1921), is helpful in partitioning the correlation coefficient into direct and indirect effects and in the assessment of relative contribution of each component to the yield. The present study was envisaged with the objective to study the character association in early segregating populations of mustard crosses.

\section{Materials and Methods}

The materials used were developed and maintained by Regional Research Station Programme on Mustard, Uttar Banga Krishi
Viswavidyalaya, Pundibari, Cooch Behar, West Bengal. The field experiments were conducted at Instructional Farm, Uttar Banga Krishi Viswavidyalaya, Pundibari, Cooch Behar, West Bengal, India, during rabi seasons of two consecutive years (2010-11 and 2011-12). The materials used were developed and maintained by Regional Research Station Programme on Mustard, Uttar Banga Krishi Viswavidyalaya, Pundibari, Cooch Behar, West Bengal. The materials represented the seeds of segregating mustard crosses which were advanced by bulk method of breeding for the individual crosses (Table 1). The experimental site belongs to the sub-tropical humid climate, being situated just south of the tropic of cancer (Table 2). The mustard crosses in their $\mathrm{F}_{3}$ generation, was sown on $30^{\text {th }}$ November $2010-11$ in the first year and the $\mathrm{F}_{4}$ generation was sown on $29^{\text {th }}$ November 2011-12 in the second year, for experimental trials. Randomized Block Design was followed for the two experiments, where segregating populations of mustard were sown with $10 \mathrm{~cm}$ plant to plant and $30 \mathrm{~cm}$ row to row spacing in $20 \mathrm{~m}^{2}$ plots, in three replications. Observations were recorded for the following characters for both the experimental trials in 2010-11 and 2011-12 i.e., plant height, height upto first branching, days to $50 \%$ flowering, days to physiological maturity, primary branches per plant, secondary branches per plant, siliquae per plant seeds per siliqua, total Chlorophyll Content, 100-seed weight and seed yield per plant.

The genotypic coefficient of variation (GCV) and phenotypic coefficient of variation (PCV), heritability in broad sense $\left(\mathrm{h}^{2}\right)$, GA as $\%$ of mean, correlation coefficient at genotypic and phenotypic level and path coefficient analysis were computed using standard statistical methods. Heritability (BS) was estimated according to Hanson et al., (1956). Phenotypic and genotypic coefficient of variation were 
estimated as per Burton (1952). GA as \% of mean was estimated according to Johnson et al., (1995). Correlations were worked out according to the procedure of Weber and Moorthy (1952). The partitioning of genotypic correlation coefficient of traits into direct and indirect effect was carried out using procedure suggested by Dewey and Lu (1959).

\section{Statistical analysis}

The statistical analysis was carried out using the software Windowstat (earlier Indostat).

\section{Results and Discussion}

Analysis of variance (ANOVA) was done with respect to each of the eleven yield attributing characters in segregating $\mathrm{F}_{3}$ population in the first year (2010-11) and $\mathrm{F}_{4}$ population in the second year (2011-12). The ANOVA (Table 3) revealed that the fifteen crosses in $F_{3}$ population in the first year except seeds per siliqua and 100 seed weight in the $F_{3}$ population in first year and $F_{4}$ population in the second year, differed significantly for all the characters. Similar findings were reported by Prasad et al., (2010) and Singh et al., (2010).

The mean performance of the $\mathrm{F}_{3}$ and $\mathrm{F}_{4}$ generations of mustard crosses revealed a lot of variability for the different yield attributing characters (Table 4). The estimates of various genetic parameters exhibited wide range of variability for all the characters (Table 5). The degree of variability shown by the different characters can be judged by the values of genotypic coefficient of variation and phenotypic coefficient of variation. The GCV and PCV were comparatively high for the character seeds per siliqua in $\mathrm{F}_{3}$ generation and height up to first fruiting branch in $\mathrm{F}_{4}$ generation which indicated the presence of high amount of both genotypic as well as phenotypic variability for these characters in the genetic material. Similar result was obtained by Uddin et al., (1995), Meena et al., (2000), Verma et al., (2001), Sudan et al., (2004), Nigam et al., (2009), Singh et al., (2011), Shazia et al., (2011), Yadav et al., (2012) Shekhawat et al., (2014) and Meena et al., (2017). The estimates of GCV and PCV were low for days to $50 \%$ flowering, days to physiological maturity and 100 seed weight in both $\mathrm{F}_{3}$ and $\mathrm{F}_{4}$ generation (Yadav et al., 2012). A close proximity in PCV and GCV was observed in plant height, height up to first fruiting branch, days to $50 \%$ flowering, days to physiological maturity and siliquae per plant in both the generations except siliquae per plant in $\mathrm{F}_{3}$ generation indicating little influence of the environment in the expression of these yield attributing characters studied. Similar results were obtained by Singh et al., (2015) and Srivastava et al., (2016).

The high estimate of $\mathrm{h}^{2}$ was observed in plant height, height up to first fruiting branch, days to $50 \%$ flowering, days to physiological maturity, and seed yield per plant, in both $\mathrm{F}_{3}$ and $\mathrm{F}_{4}$ generations, but secondary branches per plant and siliquae per plant only in $\mathrm{F}_{4}$ generation showed high heritability.

The heritability estimates for different characters depend on genetic makeup of the breeding material studied. High heritability will be effective being less influenced by environmental useful in indicating the relative value of selection based on phenotypic expression of different characters.

Thus, these characters indicated that simple selection on the basis of phenotypic performance of the genotype would be more efficient in further improvement of these characters. High heritability estimates for most of the characters studied have been reported earlier also by Diwakar and Singh (1993),Sangwan et al., (1994), Singh et al., (2013) and Vermai et al., (2016). 
Table.1 List of 15 mustard crosses evaluated over two years ( $F_{3}$ during 2010-11 and $F_{4}$ during 2011-12)

\begin{tabular}{|c|l|}
\hline Cross No. & Name of Cross \\
\hline 1 & Varuna $\times$ Bhagirathi \\
\hline 2 & PusaBahar $\times$ Rajasthan Local Sel-2 \\
\hline 3 & Varuna $\times$ Pusa Barani \\
\hline 4 & Raj Local Sel1 $\times$ Pusa Jaikissan \\
\hline 5 & Varuna $\times$ Raj Local sel-2 \\
\hline 6 & Pusa Bahar $\times$ Chaita Local \\
\hline 7 & Seeta (B-85) $\times$ Kranti \\
\hline 8 & Pusa Bold $\times$ Rajasthan Local Sel -1 \\
\hline 9 & Pusa Bahar $\times$ Rajasthan Local Sel-1 \\
\hline 10 & Pusa Bold $\times$ Seeta (B- 85) \\
\hline 11 & Seeta (B- 85) $\times$ Rajasthan Local Sel-1 \\
\hline 12 & Raj Local sel- $2 \times$ Kranti \\
\hline 13 & Raj Local sel2 $\times$ Kranti \\
\hline 14 & Raj Local sel2 $\times$ Pusa Barani \\
\hline
\end{tabular}


Table.2 Average monthly records of meteorological parameters at the experimental site i.e., instructional farm, Uttar Banga Krishi Viswavidyalaya, during rabi season of 2010-11 and 2011-12

\begin{tabular}{|c|c|c|c|c|c|c|}
\hline \multirow[t]{2}{*}{ Year } & \multirow[t]{2}{*}{ Months } & \multicolumn{2}{|c|}{ Temperature $\left({ }^{\circ} \mathrm{C}\right)$} & \multirow{2}{*}{$\begin{array}{l}\text { Total rainfall } \\
(\mathrm{mm})\end{array}$} & \multicolumn{2}{|c|}{ Relative humidity $(\%)$} \\
\hline & & Max. & Min. & & Max. & Min. \\
\hline \multirow[t]{2}{*}{2010} & November & 31.75 & 22.41 & 0.027 & 76.57 & 79.87 \\
\hline & December & 26.45 & 15.54 & 1.07 & 79.16 & 76.55 \\
\hline \multirow[t]{2}{*}{2011} & January & 24.48 & 10.65 & 0.05 & 90.29 & 70.71 \\
\hline & February & 28.18 & 12.29 & 0.031 & 82.04 & 58.89 \\
\hline \multirow[t]{2}{*}{2011} & November & 29.03 & 14.73 & 0.017 & 79.57 & 80.43 \\
\hline & December & 26.88 & 12.21 & 0.01 & 90.71 & 82.42 \\
\hline \multirow[t]{2}{*}{2012} & January & 22.39 & 9.32 & 0.11 & -99.00 & -99.00 \\
\hline & February & 26.41 & 11.48 & 0.52 & -99.00 & -99.00 \\
\hline
\end{tabular}

Source: Department of Agronomy, Uttar Banga Krishi Viswavidyalaya, Pundibari, CoochBehar, West Bengal.

Table.3 Analysis of variance for different characters of segregating populations of 15 mustard crosses

\begin{tabular}{|c|c|c|c|c|c|c|c|c|c|c|c|c|c|}
\hline \multirow[t]{2}{*}{ Year } & \multirow{2}{*}{$\begin{array}{c}\text { Sources of } \\
\text { variation }\end{array}$} & \multirow[t]{2}{*}{ d.f. } & \multicolumn{11}{|c|}{ Mean Sum of Square (MS) } \\
\hline & & & $\begin{array}{l}\text { Plant height } \\
\text { (cm) }\end{array}$ & $\begin{array}{l}\text { Height upto } \\
\text { first fruiting } \\
\text { branch }(\mathrm{cm})\end{array}$ & $\begin{array}{c}\text { Days to } \\
50 \% \\
\text { flowering }\end{array}$ & $\begin{array}{c}\text { Days to } \\
\text { physiological } \\
\text { maturity }\end{array}$ & $\begin{array}{c}\text { Primary } \\
\text { Branches } \\
\text { per plant }\end{array}$ & $\begin{array}{c}\text { Secondary } \\
\text { Branches } \\
\text { per plant }\end{array}$ & $\begin{array}{c}\text { Siliquae per } \\
\text { plant }\end{array}$ & $\begin{array}{c}\text { Seeds per } \\
\text { siliqua }\end{array}$ & $\begin{array}{c}\text { Total } \\
\text { Chlorophyll } \\
\text { content } \\
\text { (spad502) }\end{array}$ & $\begin{array}{c}100 \\
\text { seed } \\
\text { weight } \\
(\mathrm{g})\end{array}$ & $\begin{array}{l}\text { Seed } \\
\text { yield per } \\
\text { plant }(g)\end{array}$ \\
\hline \multirow{3}{*}{$\begin{array}{l}F_{3} \\
(2010-11)\end{array}$} & Replication & 2 & $46.56^{*}$ & 5.83 & 0.27 & 0.56 & 0.40 & 1.65 & 801.92 & 890.13 & $70.06^{*}$ & 0.001 & 0.36 \\
\hline & Genotypes & 14 & $693.72 * *$ & $232.66 * *$ & $14.61 * *$ & $39.70 * *$ & $0.96^{*}$ & $5.59 * *$ & $2347.86 * *$ & 1004.12 & $52.44 * *$ & 0.003 & $15.40 * *$ \\
\hline & Error & 28 & 10.47 & 11.45 & 1.65 & 1.19 & 0.45 & 1.95 & 670.67 & 866.87 & 17.74 & 0.002 & 2.47 \\
\hline \multirow{3}{*}{$\begin{array}{l}F_{4} \\
(2011-12)\end{array}$} & Replication & 2 & 0.42 & 2.76 & $8.02 *$ & 0.42 & 0.28 & 0.05 & 4.39 & 1.17 & 8.94 & 0.002 & 0.096 \\
\hline & Genotypes & 14 & $1404.84 * *$ & $664.95 * *$ & $20.02 * *$ & $38.12 * *$ & $0.83 * *$ & $8.69 * *$ & $3245.36 * *$ & $4.57 * *$ & $31.51^{* *}$ & $0.003 *$ & $15.12 * *$ \\
\hline & Error & 28 & 5.11 & 3.92 & 2.38 & 1.71 & 0.24 & 0.78 & 5.73 & 1.17 & 8.93 & 0.001 & 0.30 \\
\hline
\end{tabular}

$*, * *=$ Significant at $5 \%$ and $1 \%$ levels, respectively 
Table.4 Mean table for different characters of segregating population of 15 mustard crosses over two years

\begin{tabular}{|c|c|c|c|c|c|c|c|c|c|c|c|c|}
\hline Characters & & $\begin{array}{c}\text { Plant } \\
\text { height } \\
(\mathrm{cm})\end{array}$ & $\begin{array}{l}\text { Height upto } \\
\text { first fruiting } \\
\text { branch }(\mathrm{cm})\end{array}$ & $\begin{array}{l}\text { Days to } \\
50 \% \\
\text { flowering }\end{array}$ & $\begin{array}{c}\text { Days to } \\
\text { physiological } \\
\text { maturity }\end{array}$ & $\begin{array}{l}\text { Primary } \\
\text { Branches } \\
\text { per plant }\end{array}$ & $\begin{array}{l}\text { Secondary } \\
\text { Branches } \\
\text { per plant }\end{array}$ & $\begin{array}{l}\text { Siliquae } \\
\text { per plant }\end{array}$ & $\begin{array}{l}\text { Seeds } \\
\text { per } \\
\text { siliqua }\end{array}$ & $\begin{array}{c}\text { Total } \\
\text { Chlorophyll } \\
\text { content } \\
\text { (spad502) }\end{array}$ & $\begin{array}{l}100 \text { seed } \\
\text { weight } \\
\text { (g) }\end{array}$ & $\begin{array}{c}\text { Seed } \\
\text { Yield per } \\
\text { plant (g) }\end{array}$ \\
\hline \multirow[t]{2}{*}{ Varuna $\times$ Bhagirathi } & $\mathrm{F}_{3}(2010-11)$ & 143.53 & 27.57 & 52.00 & 94.33 & 4.87 & 7.67 & 126.07 & 13.87 & 37.67 & 0.47 & 10.75 \\
\hline & $\mathrm{F}_{4}(2011-12)$ & 147.33 & 44.50 & 51.67 & 115.33 & 4.60 & 8.10 & 217.33 & 14.03 & 43.33 & 0.44 & 7.29 \\
\hline \multirow[t]{2}{*}{ Pusa Bahar× RajLocal Sel -2 } & $\mathrm{F}_{3}(2010-11)$ & 142.80 & 25.10 & 50.33 & 98.00 & 5.37 & 6.50 & 164.17 & 13.77 & 45.93 & 0.52 & 11.61 \\
\hline & $\mathrm{F}_{4}(2011-12)$ & 152.33 & 21.83 & 51.33 & 105.33 & 5.30 & 6.20 & 284.87 & 11.17 & 43.22 & 0.47 & 10.85 \\
\hline \multirow[t]{2}{*}{ Varuna $\times$ Pusa Barani } & $\mathrm{F}_{3}(2010-11)$ & 137.33 & 19.73 & 49.33 & 95.33 & 5.23 & 5.43 & 216.57 & 13.53 & 43.27 & 0.44 & 10.36 \\
\hline & $\mathrm{F}_{4}(2011-12)$ & 174.67 & 54.27 & 53.67 & 110.00 & 4.60 & 6.87 & 227.83 & 10.63 & 42.37 & 0.52 & 8.54 \\
\hline \multirow[t]{2}{*}{ Raj Local Sel-1× Pusa Jaikissan } & $\mathrm{F}_{3}(2010-11)$ & 153.70 & 17.73 & 52.33 & 99.00 & 5.73 & 7.23 & 201.73 & 13.43 & 48.17 & 0.48 & 8.94 \\
\hline & $\mathrm{F}_{4}(2011-12)$ & 177.30 & 56.83 & 53.33 & 110.00 & 5.70 & 10.33 & 234.50 & 13.10 & 48.27 & 0.48 & 10.27 \\
\hline \multirow[t]{2}{*}{ Varuna $\times$ Raj Local sel- 2} & $\mathrm{~F}_{3}(2010-11)$ & 125.80 & 22.63 & 52.33 & 96.00 & 5.60 & 8.27 & 177.47 & 12.93 & 38.67 & 0.43 & 6.31 \\
\hline & $\mathrm{F}_{4}(2011-12)$ & 187.00 & 36.30 & 48.67 & 107.67 & 5.07 & 8.17 & 195.77 & 12.77 & 45.30 & 0.50 & 9.66 \\
\hline \multirow[t]{2}{*}{ Pusa Bahar $\times$ Chaita Local } & $\mathrm{F}_{3}(2010-11)$ & 115.34 & 17.27 & 46.67 & 95.00 & 4.47 & 4.10 & 115.33 & 19.23 & 44.70 & 0.43 & 5.21 \\
\hline & $\mathrm{F}_{4}(2011-12)$ & 142.33 & 37.40 & 55.67 & 114.33 & 4.30 & 4.77 & 172.33 & 13.83 & 49.84 & 0.42 & 5.17 \\
\hline \multirow[t]{2}{*}{ Seeta $($ B-85) $\times$ Kranti } & $\mathrm{F}_{3}(2010-11)$ & 136.80 & 35.13 & 47.00 & 98.33 & 4.33 & 8.80 & 162.93 & 12.43 & 39.30 & 0.44 & 11.64 \\
\hline & $\mathrm{F}_{4}(2011-12)$ & 150.03 & 29.20 & 50.00 & 113.00 & 5.83 & 9.08 & 238.07 & 13.33 & 40.20 & 0.46 & 8.67 \\
\hline \multirow[t]{2}{*}{ Pusa Bold $\times$ Raj Local Sel -1 } & $F_{3}(2010-11)$ & 150.37 & 33.90 & 50.00 & 104.67 & 4.53 & 7.53 & 177.90 & 32.60 & 44.53 & 0.45 & 12.28 \\
\hline & $\mathrm{F}_{4}(2011-12)$ & 108.53 & 20.43 & 52.00 & 114.67 & 4.17 & 7.10 & 193.37 & 13.63 & 42.90 & 0.53 & 9.22 \\
\hline \multirow[t]{2}{*}{ Pusa Bahar $\times$ Raj Local Sel- 1} & $\mathrm{~F}_{3}(2010-11)$ & 164.70 & 71.03 & 47.67 & 103.33 & $5 . .50$ & 8.70 & 203.20 & 12.90 & 34.50 & 0.51 & 13.62 \\
\hline & $\mathrm{F}_{4}(2011-12)$ & 155.07 & 22.60 & 53.00 & 114.00 & 4.27 & 8.47 & 227.20 & 12.20 & 43.67 & 0.48 & 7.51 \\
\hline \multirow[t]{2}{*}{ Pusa Bold $\times$ Seeta (B- 85) } & $\mathrm{F}_{3}(2010-11)$ & 165.60 & 25.37 & 48.00 & 97.33 & 5.67 & 7.50 & 157.23 & 14.10 & 41.33 & 0.50 & 10.50 \\
\hline & $\mathrm{F}_{4}(2011-12)$ & 156.16 & 15.73 & 51.67 & 111.67 & 4.80 & 9.13 & 206.00 & 12.50 & 37.40 & 0.46 & 11.27 \\
\hline \multirow[t]{2}{*}{ Seeta (B- 85)×RajLocal Sel- 1} & $\mathrm{~F}_{3}(2010-11)$ & 140.30 & 35.10 & 53.00 & 93.33 & 5.97 & 6.93 & 195.63 & 11.90 & 38.60 & 0.44 & 10.57 \\
\hline & $\mathrm{F}_{4}(2011-12)$ & 155.70 & 25.53 & 52.33 & 117.67 & 5.63 & 8.70 & 153.00 & 15.30 & 38.43 & 0.50 & 12.27 \\
\hline \multirow[t]{2}{*}{ Raj Local sel- $2 \times$ Kranti } & $\mathrm{F}_{3}(2010-11)$ & 156.07 & 52.50 & 46.33 & 94.60 & 4.53 & 7.00 & 165.13 & 11.77 & 35.73 & 0.47 & 9.76 \\
\hline & $\mathrm{F}_{4}(2011-12)$ & 132.47 & 15.20 & 49.00 & 115.00 & 5.03 & 7.93 & 222.63 & 14.15 & 44.83 & 0.43 & 11.29 \\
\hline \multirow[t]{2}{*}{ Raj Local sel- 2 × Pusa Barani } & $\mathrm{F}_{3}(2010-11)$ & 125.63 & 28.10 & 48.00 & 98.33 & 4.27 & 6.70 & 159.83 & 13.20 & 39.63 & 0.46 & 11.43 \\
\hline & $\mathrm{F}_{4}(2011-12)$ & 171.83 & 55.50 & 53.67 & 115.00 & 4.70 & 3.83 & 171.83 & 12.57 & 42.90 & 0.48 & 12.53 \\
\hline \multirow[t]{2}{*}{ Raj Local sel- $1 \times$ Kranti } & $\mathrm{F}_{3}(2010-11)$ & 130.60 & 25.47 & 50.00 & 104.33 & 4.80 & 8.13 & 164.33 & 11.00 & 47.53 & 0.49 & 10.64 \\
\hline & $\mathrm{F}_{4}(2011-12)$ & 146.37 & 35.20 & 57.00 & 117.00 & 4.80 & 7.70 & 201.33 & 13.17 & 45.30 & 0.47 & 6.96 \\
\hline \multirow[t]{2}{*}{ Seeta (B- 85) $\times$ Raj Local Sel- 2} & $\mathrm{~F}_{3}(2010-11)$ & 123.10 & 29.80 & 49.00 & 96.00 & 4.83 & 4.80 & 195.53 & 12.23 & 41.70 & 0.48 & 7.38 \\
\hline & $\mathrm{F}_{4}(2011-12)$ & 115.60 & 14.57 & 47.33 & 109.00 & 4.63 & 8.60 & 236.47 & 14.60 & 43.20 & 0.46 & 6.22 \\
\hline \multicolumn{2}{|l|}{ Mean $\left(F_{3}\right)$} & 140.78 & 28.43 & 49.47 & 97.86 & 5.05 & 7.02 & 172.20 & 19.26 & 41.42 & 0.47 & 10.07 \\
\hline \multicolumn{2}{|l|}{ Mean $\left(\mathbf{F}_{4}\right)$} & 151.51 & 32.34 & 52.02 & 112.64 & 4.90 & 7.66 & 212.17 & 13.13 & 43.41 & 0.47 & 9.18 \\
\hline \multicolumn{2}{|l|}{$C D$ of $F_{3}(P=0.05)$} & 5.41 & 5.66 & 2.15 & 1.83 & 1.12 & 2.33 & 43.31 & - & 7.04 & - & 2.62 \\
\hline \multicolumn{2}{|l|}{$C D$ of $F_{4}(P=0.05)$} & 3.78 & 3.31 & 2.58 & 2.19 & 0.82 & 1.48 & 4.00 & 1.81 & 5.00 & 0.06 & 0.92 \\
\hline
\end{tabular}




\section{Int.J.Curr.Microbiol.App.Sci (2018) 7(3): 198-211}

Table.5 Genetic parameters for different characters of segregating population of 15 mustard crosses over two years

\begin{tabular}{|c|c|c|c|c|c|c|c|c|c|c|c|c|}
\hline \multicolumn{2}{|c|}{ Characters } & $\begin{array}{l}\text { Plant height } \\
\text { (cm) }\end{array}$ & $\begin{array}{l}\text { Height } \\
\text { upto first } \\
\text { fruiting } \\
\text { branch } \\
\text { (cm) }\end{array}$ & $\begin{array}{l}\text { Days to } \\
50 \% \\
\text { flowering }\end{array}$ & $\begin{array}{c}\text { Days to } \\
\text { physiologica } \\
\text { I maturity }\end{array}$ & $\begin{array}{l}\text { Primary } \\
\text { Branches } \\
\text { per plant }\end{array}$ & $\begin{array}{l}\text { Secondary } \\
\text { Branches } \\
\text { per plant }\end{array}$ & $\begin{array}{l}\text { Siliquae per } \\
\text { plant }\end{array}$ & $\begin{array}{l}\text { Seeds per } \\
\text { siliqua }\end{array}$ & $\begin{array}{c}\text { Total } \\
\text { Chlorophyl } \\
\text { I content } \\
\text { (spad502) }\end{array}$ & $\begin{array}{l}100 \text { seed } \\
\text { weight } \\
(\mathrm{g})\end{array}$ & $\begin{array}{l}\text { Seed } \\
\text { Yield per } \\
\text { plant (g) }\end{array}$ \\
\hline \multirow[t]{2}{*}{ Mean } & $\mathrm{F}_{3}(2010-11)$ & 140.78 & 28.43 & 49.47 & 97.86 & 5.05 & 7.02 & 172.20 & 19.26 & 41.42 & 0.47 & 10.07 \\
\hline & $\mathrm{F}_{4}(2011-12)$ & 151.51 & 32.34 & 52.02 & 112.64 & 4.90 & 7.67 & 212.17 & 13.13 & 43.41 & 0.47 & 9.18 \\
\hline \multirow[t]{2}{*}{$\begin{array}{l}\text { Rang } \\
\text { e }\end{array}$} & $\mathrm{F}_{3}(2010-11)$ & $\begin{array}{l}112.23- \\
169.70\end{array}$ & $13.70-55.50$ & $\begin{array}{l}44.00- \\
53.00\end{array}$ & $92.00-107.00$ & $3.20-6.80$ & $3.60-10.90$ & $114.0-283.20$ & $\begin{array}{c}8.90- \\
210.90\end{array}$ & $29.80-52.30$ & $0.35-0.61$ & $\begin{array}{l}4.75- \\
14.33\end{array}$ \\
\hline & $\mathrm{F}_{4}(2011-12)$ & $\begin{array}{c}107.60- \\
189.00\end{array}$ & $13.10-58.00$ & $45.00-58.00$ & $\begin{array}{c}104.00- \\
119.00\end{array}$ & $3.90-7.00$ & $3.10-11.90$ & $\begin{array}{l}152.00- \\
289.00\end{array}$ & $\begin{array}{c}10.00- \\
17.10\end{array}$ & $34.30-52.30$ & $0.34-0.54$ & $\begin{array}{l}4.900- \\
13.43\end{array}$ \\
\hline \multirow{2}{*}{$\begin{array}{l}\mathrm{CV} \\
(\%)\end{array}$} & $\mathrm{F}_{3}(2010-11)$ & 2.30 & 11.90 & 2.59 & 1.12 & 13.30 & 19.89 & 15.04 & 152.87 & 10.17 & 9.05 & 15.61 \\
\hline & $\mathrm{F}_{4}(2011-12)$ & 1.49 & 6.12 & 2.96 & 1.16 & 10.03 & 11.52 & 1.13 & 8.25 & 6.88 & 7.32 & 9.10 \\
\hline \multirow[t]{2}{*}{$\mathbf{P C V}$} & $\mathrm{F}_{3}(2010-11)$ & 10.96 & 32.47 & 4.94 & 3.83 & 15.60 & 25.34 & 20.36 & 156.85 & 13.07 & 9.79 & 25.86 \\
\hline & $\mathrm{F}_{4}(2011-12)$ & 14.33 & 46.31 & 5.52 & 3.30 & 13.50 & 24.11 & 15.53 & 11.56 & 9.34 & 8.64 & 24.94 \\
\hline \multirow[t]{2}{*}{ GCV } & $\mathrm{F}_{3}(2010-11)$ & 10.72 & 30.20 & 4.20 & 3.66 & 8.15 & 15.70 & 13.73 & 35.12 & 8.21 & 3.74 & 20.62 \\
\hline & $\mathrm{F}_{4}(2011-12)$ & 14.26 & 45.90 & 4.66 & 3.09 & 9.04 & 21.18 & 15.49 & 8.10 & 6.32 & 4.59 & 24.21 \\
\hline \multirow{2}{*}{$\begin{array}{l}h^{2} \\
(B . S)\end{array}$} & $\mathrm{F}_{3}(2010-11)$ & 95.6 & 86.6 & 72.4 & 91.5 & 27.3 & 38.4 & 45.5 & 5.00 & 39.5 & 14.6 & 63.6 \\
\hline & $\mathrm{F}_{4}(2011-12)$ & 98.9 & 98.3 & 71.2 & 87.7 & 44.8 & 77.2 & 99.5 & 49.1 & 45.7 & 28.2 & 94.2 \\
\hline \multirow{2}{*}{$\begin{array}{l}\text { GA } \\
\text { as \% } \\
\text { of } \\
\text { mean }\end{array}$} & $\mathrm{F}_{3}(2010-11)$ & 21.59 & 57.89 & 7.36 & 7.21 & 8.77 & 20.03 & 19.07 & 16.20 & 10.63 & 2.95 & 33.88 \\
\hline & $\mathrm{F}_{4}(2011-12)$ & 29.21 & 93.72 & 8.10 & 5.96 & 12.46 & 38.33 & 31.82 & 11.69 & 8.80 & 5.02 & 48.40 \\
\hline
\end{tabular}


Table.6 Genotypic association between yield and its attributing traits in segregating population of 15 mustard crosses over two years

\begin{tabular}{|c|c|c|c|c|c|c|c|c|c|c|c|c|}
\hline $\begin{array}{l}\text { Sl. } \\
\text { No }\end{array}$ & Characters & & $\begin{array}{l}\text { Height } \\
\text { upto first } \\
\text { fruiting } \\
\text { branch } \\
\text { (cm) }\end{array}$ & $\begin{array}{c}\text { Days to } \\
50 \% \\
\text { flowering }\end{array}$ & $\begin{array}{c}\text { Days to } \\
\text { physiological } \\
\text { maturity }\end{array}$ & $\begin{array}{l}\text { Primary } \\
\text { Branches } \\
\text { per plant }\end{array}$ & $\begin{array}{c}\text { Secondary } \\
\text { Branches } \\
\text { per plant }\end{array}$ & $\begin{array}{l}\text { Siliquae } \\
\text { per plant }\end{array}$ & $\begin{array}{l}\text { Seeds per } \\
\text { siliqua }\end{array}$ & $\begin{array}{l}\text { Total } \\
\text { Chlorophy } \\
\text { ll content } \\
\text { (spad,502) }\end{array}$ & $\begin{array}{l}100 \text { seed } \\
\text { weight }(\mathrm{g})\end{array}$ & $\begin{array}{c}\text { Seed Yield } \\
\text { per plant } \\
\text { (g) }\end{array}$ \\
\hline \multirow[t]{2}{*}{1} & \multirow[t]{2}{*}{ Plant height $(\mathrm{cm})$} & $\mathrm{F}_{3}(2010-11)$ & 0.354 & -0.173 & 0.275 & $0.560 *$ & $0.617 * *$ & 0.317 & $-0.975 * *$ & -0.302 & $0.913 * *$ & $0.669 * *$ \\
\hline & & $\mathrm{F}_{4}(2011-12)$ & $0.661 * *$ & 0.005 & -0.277 & $0.460 *$ & 0.027 & -0.053 & $-0.580 *$ & 0.066 & 0.264 & 0.355 \\
\hline \multirow[t]{2}{*}{2} & \multirow{2}{*}{$\begin{array}{l}\text { Height upto first fruiting } \\
\text { branch }(\mathrm{cm})\end{array}$} & $\mathrm{F}_{3}(2010-11)$ & & -0.356 & -0.037 & -0.354 & 0.377 & 0.045 & $-0.823 * *$ & $-0.746^{* *}$ & 0.056 & 0.399 \\
\hline & & $\mathrm{F}_{4}(2011-12)$ & & $0.529 *$ & 0.037 & 0.108 & -0.266 & -0.169 & -0.383 & $0.474^{*}$ & 0.245 & 0.007 \\
\hline \multirow[t]{2}{*}{3} & \multirow[t]{2}{*}{ Days to $50 \%$ flowering } & $\mathrm{F}_{3}(2010-11)$ & & & -0.014 & $0.985 * *$ & 0.124 & 0.341 & $-0.908 * *$ & 0.359 & -0.049 & -0.141 \\
\hline & & $\mathrm{F}_{4}(2011-12)$ & & & $0.489 *$ & -0.313 & -0.425 & -0.365 & -0.227 & 0.380 & 0.122 & -0.191 \\
\hline \multirow[t]{2}{*}{4} & \multirow{2}{*}{$\begin{array}{l}\text { Days to physiological } \\
\text { maturity }\end{array}$} & $\mathrm{F}_{3}(2010-11)$ & & & -0.093 & -0.226 & $0.629 * *$ & 0.213 & -0.012 & 0.350 & $0.587 *$ & $0.560 *$ \\
\hline & & $\mathrm{F}_{4}(2011-12)$ & & & & -0.252 & -0.102 & $-0.688 * *$ & $0.660 * *$ & -0.120 & -0.009 & -0.012 \\
\hline \multirow[t]{2}{*}{5} & \multirow{2}{*}{$\begin{array}{l}\text { Primary Branches per } \\
\text { plant }\end{array}$} & $\mathrm{F}_{3}(2010-11)$ & & & & & 0.397 & $0.624 * *$ & $-0.914 * *$ & -0.052 & $0.485^{*}$ & 0.150 \\
\hline & & $\mathrm{F}_{4}(2011-12)$ & & & & & $0.511 *$ & 0.259 & 0.091 & -0.239 & -0.009 & $0.603 * *$ \\
\hline \multirow[t]{2}{*}{6} & \multirow{2}{*}{$\begin{array}{l}\text { Secondary Branches per } \\
\text { plant }\end{array}$} & $\mathrm{F}_{3}(2010-11)$ & & & & & & 0.100 & $-0.935 * *$ & $-0.502 *$ & 0.362 & $0.688 * *$ \\
\hline & & $\mathrm{F}_{4}(2011-12)$ & & & & & & 0.291 & 0.313 & -0.297 & 0.042 & 0.016 \\
\hline \multirow[t]{2}{*}{7} & \multirow[t]{2}{*}{ Siliquae per plant } & $\mathrm{F}_{3}(2010-11)$ & & & & & & & $-0.949 * *$ & -0.010 & -0.046 & 0.235 \\
\hline & & $\mathrm{F}_{4}(2011-12)$ & & & & & & & $-0.564^{*}$ & 0.050 & -0.205 & -0.121 \\
\hline \multirow[t]{2}{*}{8} & \multirow[t]{2}{*}{ Seeds per siliqua } & $\mathrm{F}_{3}(2010-11)$ & & & & & & & & $0.811 * *$ & $-0.997 * *$ & $-0.939 * *$ \\
\hline & & $\mathrm{F}_{4}(2011-12)$ & & & & & & & & 0.090 & $-0.524^{*}$ & -0.138 \\
\hline \multirow[t]{2}{*}{9} & \multirow{2}{*}{$\begin{array}{l}\text { Total Chlorophyll content } \\
\text { (spad }\end{array}$} & $\mathrm{F}_{3}(2010-11)$ & & & & & & & & & 0.025 & -0.252 \\
\hline & & $\mathrm{F}_{4}(2011-12)$ & & & & & & & & & $-0.473 *$ & $-0.560 *$ \\
\hline \multirow[t]{2}{*}{10} & \multirow[t]{2}{*}{100 seed weight $(\mathrm{g})$} & $\mathrm{F}_{3}(2010-11)$ & & & & & & & & & & $0.819 * *$ \\
\hline & & $\mathrm{F}_{4}(2011-12)$ & & & & & & & & & & 0.388 \\
\hline
\end{tabular}

\footnotetext{
$*, * *=$ Significant at $5 \%$ and $1 \%$ levels, respectively
} 


\section{Int.J.Curr.Microbiol.App.Sci (2018) 7(3): 198-211}

Table.7 Phenotypic association between yield and its attributing traits in segregating population of 15 mustard crosses over two years

\begin{tabular}{|c|c|c|c|c|c|c|c|c|c|c|c|c|}
\hline $\begin{array}{l}\text { Sl. } \\
\text { No. }\end{array}$ & Characters & & $\begin{array}{l}\text { Height upto } \\
\text { first fruiting } \\
\text { branch (cm) }\end{array}$ & $\begin{array}{l}\text { Days to } \\
50 \% \\
\text { flowering }\end{array}$ & $\begin{array}{c}\text { Days to } \\
\text { physiologica } \\
\text { I maturity }\end{array}$ & $\begin{array}{l}\text { Primary } \\
\text { Branches } \\
\text { per plant }\end{array}$ & $\begin{array}{l}\text { Secondary } \\
\text { Branches } \\
\text { per plant }\end{array}$ & $\begin{array}{l}\text { Siliquae } \\
\text { per plant }\end{array}$ & $\begin{array}{l}\text { Seeds } \\
\text { per } \\
\text { siliqua }\end{array}$ & $\begin{array}{c}\text { Total } \\
\text { Chloroph } \\
\text { yll content } \\
\text { (spad,502) }\end{array}$ & $\begin{array}{l}100 \text { seed } \\
\text { weight } \\
(\mathrm{g})\end{array}$ & $\begin{array}{c}\text { Seed } \\
\text { Yield } \\
\text { per } \\
\text { plant (g) }\end{array}$ \\
\hline \multirow[t]{2}{*}{1} & \multirow[t]{2}{*}{ Plant height $(\mathrm{cm})$} & $\mathrm{F}_{3}(2010-11)$ & 0.320 & -0.054 & 0.258 & 0.288 & 0.400 & 0.234 & -0.195 & -0.187 & 0.392 & $0.536 *$ \\
\hline & & $\mathrm{F}_{4}(2011-12)$ & $0.655 * *$ & 0.175 & -0.262 & 0.293 & 0.011 & -0.054 & -0.402 & 0.029 & 0.141 & 0.338 \\
\hline \multirow[t]{2}{*}{2} & \multirow{2}{*}{$\begin{array}{l}\text { Height upto first } \\
\text { fruiting branch }(\mathrm{cm})\end{array}$} & $\mathrm{F}_{3}(2010-11)$ & & -0.266 & -0.054 & -0.258 & 0.197 & 0.017 & -0.141 & $-0.451 *$ & 0.092 & 0.303 \\
\hline & & $\mathrm{F}_{4}(2011-12)$ & & 0.432 & 0.015 & 0.052 & -0.241 & -0.167 & -0.224 & 0.272 & 0.116 & 0.007 \\
\hline \multirow[t]{2}{*}{3} & \multirow[t]{2}{*}{ Days to $50 \%$ flowering } & $\mathrm{F}_{3}(2010-11)$ & & & -0.113 & 0.317 & 0.252 & 0.182 & -0.139 & 0.094 & 0.002 & 0.030 \\
\hline & & $\mathrm{F}_{4}(2011-12)$ & & & 0.371 & -0.162 & -0.290 & -0.306 & -0.246 & 0.201 & 0.033 & -0.165 \\
\hline \multirow[t]{2}{*}{4} & \multirow{2}{*}{$\begin{array}{l}\text { Days to physiological } \\
\text { maturity }\end{array}$} & $\mathrm{F}_{3}(2010-11)$ & & & & -0.073 & 0.311 & 0.158 & 0.013 & 0.261 & 0.205 & $0.460 *$ \\
\hline & & $\mathrm{F}_{4}(2011-12)$ & & & & -0.097 & -0.079 & $-0.655 * *$ & 0.412 & -0.092 & -0.158 & -0.042 \\
\hline \multirow[t]{2}{*}{5} & \multirow{2}{*}{$\begin{array}{l}\text { Primary Branches per } \\
\text { plant }\end{array}$} & $\mathrm{F}_{3}(2010-11)$ & & & & & 0.027 & 0.418 & -0.114 & 0.061 & 0.176 & -0.105 \\
\hline & & $\mathrm{F}_{4}(2011-12)$ & & & & & 0.429 & 0.162 & 0.150 & -0.189 & -0.049 & 0.352 \\
\hline \multirow[t]{2}{*}{6} & \multirow{2}{*}{$\begin{array}{l}\text { Secondary Branches } \\
\text { per plant }\end{array}$} & $\mathrm{F}_{3}(2010-11)$ & & & & & & 0.108 & -0.153 & -0.188 & 0.167 & $0.463^{*}$ \\
\hline & & $\mathrm{F}_{4}(2011-12)$ & & & & & & 0.260 & 0.176 & -0.203 & 0.055 & 0.026 \\
\hline \multirow[t]{2}{*}{7} & \multirow[t]{2}{*}{ Siliquae per plant } & $\mathrm{F}_{3}(2010-11)$ & & & & & & & -0.209 & 0.004 & 0.098 & 0.273 \\
\hline & & $\mathrm{F}_{4}(2011-12)$ & & & & & & & -0.385 & 0.032 & -0.095 & -0.102 \\
\hline \multirow[t]{2}{*}{8} & \multirow[t]{2}{*}{ Seeds per siliqua } & $\mathrm{F}_{3}(2010-11)$ & & & & & & & & 0.182 & -0.008 & -0.251 \\
\hline & & $\mathrm{F}_{4}(2011-12)$ & & & & & & & & -0.088 & -0.161 & -0.043 \\
\hline \multirow[t]{2}{*}{9} & \multirow{2}{*}{$\begin{array}{l}\text { Total Chlorophyll } \\
\text { content (spad }\end{array}$} & $\mathrm{F}_{3}(2010-11)$ & & & & & & & & & 0.040 & -0.184 \\
\hline & & $\mathrm{F}_{4}(2011-12)$ & & & & & & & & & -0.158 & -0.374 \\
\hline \multirow[t]{2}{*}{10} & \multirow[t]{2}{*}{100 seed weight $(\mathrm{g})$} & $\mathrm{F}_{3}(2010-11)$ & & & & & & & & & & 0.278 \\
\hline & & $\mathrm{F}_{4}(2011-12)$ & & & & & & & & & & 0.205 \\
\hline
\end{tabular}

$*, * *=$ Significant at $5 \%$ and $1 \%$ levels, respectively 
Table.8 Genotypic direct (diagonal) and indirect (off-diagonal) effects of different attributing triaits on seed yield in segregating population of 15 mustard crosses over two years

\begin{tabular}{|c|c|c|c|c|c|c|c|c|c|c|c|c|c|}
\hline $\begin{array}{l}\text { Sl. } \\
\text { No. }\end{array}$ & Characters & & $\begin{array}{l}\text { Plant } \\
\text { height } \\
(\mathrm{cm})\end{array}$ & $\begin{array}{l}\text { Height } \\
\text { upto first } \\
\text { fruiting } \\
\text { branch } \\
\text { (cm) }\end{array}$ & $\begin{array}{l}\text { Days to } \\
50 \% \\
\text { floweri } \\
\text { ng }\end{array}$ & $\begin{array}{c}\text { Days to } \\
\text { physiologi } \\
\text { cal } \\
\text { maturity }\end{array}$ & $\begin{array}{c}\text { Primar } \\
\text { y } \\
\text { Branch } \\
\text { es per } \\
\text { plant }\end{array}$ & $\begin{array}{l}\text { Secondar } \\
\text { y } \\
\text { Branches } \\
\text { per plant }\end{array}$ & $\begin{array}{l}\text { Siliqua } \\
\text { e per } \\
\text { plant }\end{array}$ & $\begin{array}{l}\text { Seeds } \\
\text { per } \\
\text { siliqua }\end{array}$ & $\begin{array}{l}\text { Total } \\
\text { Chlorophy } \\
\text { Il content } \\
\text { (spad502) }\end{array}$ & $\begin{array}{l}100 \text { seed } \\
\text { weight } \\
\text { (g) }\end{array}$ & $\begin{array}{c}\text { Correlat } \\
\text { ion with } \\
\text { Seed } \\
\text { Yield } \\
\text { per } \\
\text { plant (g) }\end{array}$ \\
\hline \multirow[t]{2}{*}{1} & \multirow[t]{2}{*}{ Plant height $(\mathrm{cm})$} & $\mathrm{F}_{3}(2010-11)$ & 1.04 & 0.17 & 0.01 & -0.02 & -0.07 & 0.50 & -0.01 & -0.08 & -0.34 & -0.53 & $0.67 *$ \\
\hline & & $\mathrm{F}_{4}(2011-12)$ & -0.28 & 4.70 & 1.34 & 2.89 & -0.13 & 0.08 & 0.31 & -0.11 & -0.61 & -1.83 & 0.36 \\
\hline \multirow[t]{2}{*}{2} & \multirow{2}{*}{$\begin{array}{l}\text { Height upto first } \\
\text { fruiting branch } \\
(\mathrm{cm})\end{array}$} & $\mathrm{F}_{3}(2010-11)$ & 0.37 & 0.47 & 0.12 & 0.00 & 0.05 & 0.31 & 0.00 & -0.04 & -0.83 & -0.04 & 0.40 \\
\hline & & $\mathrm{F}_{4}(2011-12)$ & -4.15 & 7.11 & 3.15 & -0.14 & -0.03 & -0.77 & 0.99 & -0.07 & -4.38 & -1.70 & 0.01 \\
\hline \multirow[t]{2}{*}{3} & \multirow{2}{*}{$\begin{array}{l}\text { Days to } 50 \% \\
\text { flowering }\end{array}$} & $\mathrm{F}_{3}(2010-11)$ & -0.02 & -0.17 & -0.33 & 0.01 & -0.13 & 0.10 & -0.01 & -0.03 & 0.40 & 0.03 & -0.14 \\
\hline & & $\mathrm{F}_{4}(2011-12)$ & -1.42 & 3.77 & 5.95 & -5.10 & 0.09 & -1.22 & 2.14 & -0.04 & -3.51 & -0.85 & -0.19 \\
\hline \multirow[t]{2}{*}{4} & \multirow{2}{*}{$\begin{array}{l}\text { Days to } \\
\text { physiological } \\
\text { maturity }\end{array}$} & $\mathrm{F}_{3}(2010-11)$ & 0.29 & -0.02 & 0.03 & -0.08 & 0.03 & 0.51 & 0.00 & -0.25 & 0.39 & -0.34 & $0.56^{*}$ \\
\hline & & $\mathrm{F}_{4}(2011-12)$ & 1.74 & 0.10 & 2.91 & -10.41 & 0.07 & -0.29 & 4.03 & 0.12 & 1.11 & 0.61 & -0.01 \\
\hline 5 & $\begin{array}{l}\text { Primary Branches } \\
\text { per plant }\end{array}$ & $\mathrm{F}_{4}(2011-12)$ & -2.89 & 0.77 & -1.86 & 2.63 & -0.29 & 1.47 & -1.52 & 0.02 & 2.21 & 0.07 & $0.60 * *$ \\
\hline \multirow[t]{2}{*}{6} & \multirow{2}{*}{$\begin{array}{l}\text { Secondary } \\
\text { Branches per plant }\end{array}$} & $\mathrm{F}_{3}(2010-11)$ & 0.64 & 0.18 & -0.04 & -0.05 & -0.05 & 0.81 & 0.00 & -0.03 & -0.56 & -0.21 & $0.69 * *$ \\
\hline & & $\mathrm{F}_{4}(2011-12)$ & -0.17 & -1.89 & -1.53 & 1.06 & -0.15 & 2.88 & -1.70 & 0.06 & 2.74 & -0.29 & 0.02 \\
\hline \multirow[t]{2}{*}{7} & \multirow[t]{2}{*}{ Siliquae per plant } & $\mathrm{F}_{3}(2010-11)$ & 0.33 & 0.02 & -0.11 & -0.02 & -0.08 & 0.08 & -0.02 & 0.01 & -0.01 & 0.03 & 0.24 \\
\hline & & $\mathrm{F}_{4}(2011-12)$ & 0.33 & -1.20 & -2.17 & 7.16 & -0.07 & 0.84 & -5.85 & -0.10 & -0.46 & 1.42 & -0.12 \\
\hline \multirow[t]{2}{*}{8} & \multirow[t]{2}{*}{ Seeds per siliqua } & $\mathrm{F}_{3}(2010-11)$ & 0.16 & 0.04 & -0.02 & -0.26 & 0.05 & 0.04 & -0.67 & -0.51 & 0.02 & 0.21 & $-0.94 * *$ \\
\hline & & $\mathrm{F}_{4}(2011-12)$ & 3.64 & -2.72 & -1.35 & -6.87 & -0.03 & 0.90 & 3.30 & 0.19 & -0.83 & 3.63 & -0.14 \\
\hline \multirow[t]{2}{*}{9} & \multirow{2}{*}{$\begin{array}{l}\text { Total Chlorophyll } \\
\text { content (spad }\end{array}$} & $\mathrm{F}_{3}(2010-11)$ & -0.32 & -0.35 & -0.12 & -0.03 & 0.01 & -0.41 & 0.00 & -0.14 & 1.12 & -0.02 & -0.25 \\
\hline & & $\mathrm{F}_{4}(2011-12)$ & -0.41 & 3.37 & 2.26 & 1.25 & 0.07 & -0.86 & -0.93 & 0.02 & -9.25 & 3.28 & $-0.56^{*}$ \\
\hline \multirow[t]{2}{*}{10} & \multirow{2}{*}{$\begin{array}{l}100 \text { seed weight } \\
(\mathrm{g})\end{array}$} & $\mathrm{F}_{3}(2010-11)$ & 0.95 & 0.03 & 0.02 & -0.05 & -0.06 & 0.29 & 0.00 & 0.19 & 0.03 & -0.58 & $0.82 * *$ \\
\hline & & $\mathrm{F}_{4}(2011-12)$ & -1.66 & 1.75 & 0.73 & 0.91 & 0.00 & 0.12 & 1.20 & -0.10 & 4.37 & -6.93 & 0.39 \\
\hline
\end{tabular}

$*, * *=$ Significant at $5 \%$ and $1 \%$ levels, respectively 
The GA is also useful indicator of the progress that can be expected as a result of exercising selection on the population. The high estimates of GA expressed as percentage of mean were recorded for height up to first fruiting branch and seed yield per plant in both $\mathrm{F}_{3}$ and $\mathrm{F}_{4}$ generations. Johnson et al., (1995) impressed that heritability values along with estimates of GA were more useful than heritability alone. The $\mathrm{h}^{2}$ along with GA were higher for height up to first fruiting branch and seed yield per plant in both $\mathrm{F}_{3}$ and $\mathrm{F}_{4}$ generations indicating that these characters can be improved by simple phenotypic selection as they are more likely to be controlled by additive gene action. Similar result was reported by Sangwan et al., (1994), Uddin et al., (1995) and Akabari et al., (2015).

In the present study, the genotypic correlation coefficient was higher in magnitude than their respective phenotype correlation coefficient for most of the traits indicating depression of phenotypic expression by the environmental influence (Table 6). In $\mathrm{F}_{3}$ generation seed yield was found to be positively associated with plant height, days to physiological maturity, secondary branches per plant and 100 seed weight, on the other hand seeds per siliqua was found to be negatively associated with seed yield per plant. Plant height was positively associated with primary branches per plant, secondary branches and 100 seed weight and negatively associated with seeds per siliqua. Days to physiological maturity were positively associated with secondary branches per plant and 100 seed weight. Secondary branches per plant was however, negatively associated with total chlorophyll content. In $\mathrm{F}_{4}$ generation seed yield per plant was positively associated with primary branches per plant and negatively associated with total chlorophyll content (spad). Primary branches per plant was positively associated with only secondary branches per plant.
Similar results were reported by Arunachalam and Katiyar (1991), Zehra et al., (2009), Doddabhimappa et al., (2009), Bind et al., (2014), Bhuiyan et al., (2015), Vermai et al., (2016) and Meena et al., (2017).

In phenotypic correlation, $\mathrm{F}_{3}$ generations showed positive association of seed yield per plant with plant height, days to physiological maturity and secondary branches per plant (Table 7). These three component characters however had no positive association with other yield attributing characters. In $\mathrm{F}_{4}$ none of the yield attributing characters was positively associated with seed yield per plant which indicated that selection for these characters would be fruitless to improve seed yield.

In $\mathrm{F}_{3}$ generation the high positive direct effect (Table 8) on seed yield per plant was recorded by plant height, secondary branches per plant and total chlorophyll content (spad) and among these three characters only plant height and secondary branches per plant were positively associated with seed yield, whereas total chlorophyll content (spad) was not associated with seed yield (Table 8). Similar results were reported by Behl et al., (1992), Gosh and Mukhopadhyay (1994), Kumar and Shrivastava (2000) and Bind et al., (2014). Among the other traits which were positively associated with seed yield are days to physiological maturity, secondary branches per plant and 100 seed weight. Days to physiological maturity was positively associated with seed yield because of its superior performance through plant height, secondary branches per plant and total chlorophyll content. Positive association between 100 seed weight and seed yield was due to superior performance of 100 seed weight through plant height and secondary branches per plant. In $\mathrm{F}_{4}$ generation high direct effect on seed yield per plant was exhibited by height up to first fruiting branch, 
days to $50 \%$ flowering and secondary branches per plant but direct selection through these three component characters would be fruitless as none of them are positively associated with seed yield. However primary branches per plant which had negative direct effect on seed yield but had positive association with seed yield due to its superior performance, through days to physiological maturity, secondary branches per plant and total chlorophyll content.

\section{Acknowledgements}

The authors are grateful to the Director of Research, Uttar Banga Krishi Viswavidyalaya, Cooch Behar, West Bengal, Pin-736165, India, for the financial sanction for the present study under Regional Research Station (Terai Zone), UBKV.

\section{References}

Akabari, V. R. and Niranjan, M. 2015. Genetic variability and trait association studies in Indian mustard [Brassica Juncea (L.) Czern and Coss]. International Journal of Agricultural Sciences, 11: 35-39

Al, Jibouri, H.A., Miller, P.A., Robinson, H.F. 1958. Genetic environmental variances and covariances in an upland cotton cross of interspecific origin. Agron J. 50: 633-637.

Arunachalan, V\& Katiyar, R.K. 1991. Genetics \&breeding in oilseed Brassica in Indian Agriculture (Chopra \&Shyam Prakash) Vikas Publication House (P) Ltd. New Delhi, India pp. 86-116.

Behl, R. K., Chaudhary, B. D., Singh, R. P. and Singh, D. P. 1992. Morphophysiological determination of oil yield in Brassica juncea under dry land condition. Indian J. Gen. Pl. Breeding, 52(3): 280-284.
Bhuiyan, R. S., Islam, S., Haque, M. and Hossain S. 2015. Estimation of genotypic and phenotypic coefficients variation of yield and its contributing characters of (Brassica rapa L.). American-Eurasian Journal of Agricultural \& Environmental Sciences, 15(10): 2029-2034.

Bind, D., Singh, D. and Dwivedi, V. K. 2014. Genetic variability and character association in Indian mustard [Brassica juncia (L.) czerns and cross]. Agric. Sci. Digest., 34(3):183 - 188.

Burton, G. W. 1952. Quantitative inheritance in grasses Proc. $6^{\text {th }}$ Int Grass Land Cong., 1: 227.

Dewey, D.R., Lu, K.H. 1959. A correlation and path coefficient analysis of components of crested wheat grass seed production. Agron J. 51: 515-518.

Diwakar, M. C. and Singh, A. K. 1993. Heritability and genetic advance in segregating population of yellow seeded Indian mustard [Brassica juncea (L.) Czern and Coss]. Annals of Agril. Res., 14(2): 247-248.

Doddabhimappa R, Gangapur., Prakash, B. G., Magadum, Santosh Kumar., B. J. and Hiremath, C. P. 2012. Genetic diversity analysis of mustard (Brassica juncea L.) under protected and unprotected conditions. Bioinfolet, 9(4A): $525-530$.

Ghosh, S. K. and Mukhopadyay, D. 1994. Path analysis of yield and yield attributes of toria (Brassica rapa) as affected by date of sowing and plant density. Indian J. Agric. Sci., 64(1): 5658.

Hanson, C.H Robinson, H.G \& Comstock, R.E. 1956. Biometrical studies of yield in segregating population of Korean. Korean Agron. Journal 48: 268-277

Johnson, H.W., Robinson, H.E., Comstock, R.E. 1955. Estimation of genetic and 
environmental variability in soybean. Agron J. 47: 314-318.

Kumar, N. and Shrivastava, S. 2000. Plant ideotype of Indian mustard (Brassica juncea) for late sown condition. Indian J. Genet. \& Plant Breed., 63(4): 355.

Meena H.S, Kumar Arun, Singh VV, Meena PD, Ram Bhagirath and Kulshrestha Swarnim (2017).Genetic variability and inter-relation of seed yield with contributing traits in Indian mustard (Brassica juncea). Journal of Oilseed Brassica, 8 (2): 131-137.

Meena, S. C., Chauhan, J. S. and Yadav, J. S. 2000. Genetic variability, correlation and analysis in germplasm of Indian mustard [Brassica juncea (L.) Czern and Coss]. Ind. Jour. Pl. Genet. Resources., 13(2): 171-176.

Nigam, R., Alka and Srivastava, A. 2009. Effect of genetic variability on seed yield and oil content in Indian mustard [Brassica juncea (L.) Czern and Coss]. Asian Journal of Bio Science, 4(2): 180182.

Prasad, R., Kumar, D., Singh, S.P., Singh, R.P., Agrawal, R.K. 2010. Components of Genetic Variation for Yield Traits in Crosses of Indian Mustard [Brassica juncea (L.) Czern. \& Coss.]. Indian J Plant Genet Resour 24(1): 87-91.

Sangwan, R., Prasad, L., Singh, M. and Dixit, R. K. 1994. Analysis of heritability and genetic advances in Brassica species. $J$. Plant Sci., 14(2): 577-581.

Shazia, A., Mahmud, F., Bhuiyan, S. R. and Rahim, A. 2011 Assessment of genetic variation among advanced lines of (Brassica napus L.) Agronomski glasnik, 4(5): 4-5.

Shekhawat, N., Jadeja, G. C., Singh, J. and Ramesh. 2014. Genetic diversity analysis in relation to seed yield and its component traits in Indian mustard
[Brassica juncea (L.) Czern and Coss]. The Bioscan, 9(2): 713-717.

Singh, A., Avtar, R., Singh, D. and Kumari N. 2013. Genetic divergence for seed yield and component trait in Indian mustard [Brassica juncea (L.) Czern and Coss]. Indian Journal of Plant Sciences, 2(3): 48-51.

Singh, D., Arya, R.K., Chandra, N., Niwas, R. and Salisbury, Phillip. 2010. Genetic diversity studies in relation to seed yield and its component traits in Indian mustard (Brassica juncea L. Czern \& Cross.). J of Oilseed Brassica 1(1): 1922.

Singh, M., Tomar, A., Mishra, C. N. and Srivastava, S. B. L. 2011. Genetic parameters and character association studies in Indian mustard. Journal of Oilseed Brassica, 2(1): 35-38.

Singh, N. P., Sharma, P. K., Kumar, P., Kumar, N., Kumar, V. and Malik, V. K. 2015. Inter-relationship analysis for quantitative traits in Indian mustard [Brassica juncea (L.) Czern and Coss]. Journal of Environment and Ecology, 33(4): 1578-158.

Srivastava, K. K., Vimal, S. C., Giri, S. P. and Verma, A. K. 2016. Studies on seed quality parameters in Indian mustard [Brassica juncea (L.) Czern and Coss]. International Journal of Agricultural Sciences, 12(2): 241-244.

Sudan, R.S., Singh, S.P., Kashyap, S.C. 2004. Path analysis of yield and its components in Indian mustard (Brassica juncea (L.) Czern and Coss). Ann Agric Bio Res 9(2): 119-122.

Uddin, M. J., Chaudhary, M. A. Z. and Mai, M. F. U. 1995. Genetic variability, character association and path analysis in Indian mustard (Brassica juncea L.). Ann. Bangladesh Agric., 5(1): 51-54.

Verma, O.P., Bhajan, R, and Singh, H.P. 2001. Association among seedling and 
yield contributing traits in mustard. Curciferae Newslet 23: 49-50.

Vermai, U., Thakral, N. K. and Neeru. 2016. Genetic diversity analysis in Indian mustard [Brassica juncea (L.) Czern and Coss]. International Journal of Applied Mathematics \& Statistical Sciences (IJAMSS), 6(2): 2319-3980.

Weber, C.R \& Moorty, B.R. 1952. Heritability \& non- heritability relationship \& variability of soil content \& agronomic characters in F2 generation of soyabean crosses. Agron. J. 44: 202-209.
Wright, S. 1921. Correlation and causation. $J$ Agric Res 20: 557-585.

Yadav, R. K., Chakravarty, A., Kumar, N., Yadav, J. R. and Krishna, R. 2012. Genetic variability and component anayisis of some metric traits under sodic soil condition in Indian mustard [Brassica juncea (L.) Czern and Coss]. Prog. Agric. 12(2): 396-401.

Zehra, A. and Gulcan, K. 2009. Genetic Variation, Heritability and Path Analysis of Summer Rapeseed Cultivars. Journal of Applied Biological Sciences, 2(3): 35-39.

\section{How to cite this article:}

Suvendu Kumar Roy, Lakshmi Hijam, Moumita Chakraborty, Nagnathwar Vishal Ashokappa, Sanghamitra Rout, Vinod Ashok Kale, Bijaya Sur, Bilin Maying, Aparajita Das, Abhijit Kundu, Rupsanatan Mandal and Hossain Ali Mondal. 2018. Cause and Effect Relationship in Yield and its Attributing Traits in Early Segregating Generations of Mustard Crosses under Terai Agro-Climatic Zone of West Bengal, India. Int.J.Curr.Microbiol.App.Sci. 7(03): 198211. doi: https://doi.org/10.20546/ijcmas.2018.703.024 